\title{
Simulation of induction heating technology for the production of seamless large diameter tees
}

\author{
Iurii Murashov ${ }^{1, *}$, Vyacheslav Shestakov ${ }^{1}$, Vladimir Skornyakov ${ }^{1}$, and Irina Savelieva ${ }^{1}$ \\ ${ }^{1}$ Peter the Great St. Petersburg Polytechnic University, Polytechnicheskaya 29, Saint-Petersburg, \\ 195251, Russian Federation
}

\begin{abstract}
The article is dedicated to nonstationary simulation of induction heating technology for the production of seamless large diameter tees. A mathematical model of induction heating process representing a multiphysical (heat transfer and electromagnetism) task for technology of tees production is developed. Numerical simulation was carried out for a flat spiral inductor. The developed model was verified according to the results of experimental studies. The hydrodynamic 3D mathematical model is developed for the design of the inductor cooling system. Optimal operating modes are determined by simulation results and confirmed by experimental data. The calculation results are presented for pipes with wall thicknesses: $15 \mathrm{~mm}, 40 \mathrm{~mm}, 60 \mathrm{~mm}, 70 \mathrm{~mm}$.
\end{abstract}

\section{Introduction}

Heating technology without contact had been known for more than hundred years. It comprises a wide field of applications in metalworking and in the metal-casting industry because of its outstanding benefits [1]. There were several publications that shed light on the history of induction technique [2-7] which refer to different periods and in many cases show only "a part of history"[1]. The urgent need to design a number of induction heating installations in the pipe industry and in chemical engineering, whose power is estimated by preliminary calculations with the total capacity of the existing high-frequency electrothermal equipment in all industries, requires the development of a unified methodology for thermal and electrical calculations and the development of recommendations for the design of these technological installation [8].

Design of induction systems is a complicated process that is based on existing knowledge in different scientific fields (electromagnetism, heat transfer, material science, mechanics etc.)[1].

The heating process must be divided into the temperature intervals for an analytical solution, and the physical properties are assumed to be constant in each of the intervals [9]. Numerical methods allow one to solve separately the heat transfer equation and Maxwell's equations.

Simulation of physical processes in most cases is based on the use of numerical simulation methods at present. A corresponding software product is needed that can provide

*Corresponding author: iuriimurashov@gmail.com 
with a sufficient accuracy and efficiency a wide range of possibilities for the fastest and most qualitative construction of physical models, when solving numerical simulation problems [10-12].

The design task of induction heating installations is reduced to simulation thermal and electromagnetic processes. The aim of the simulation is to obtain the parameters of an induction heating installation without additional expensive research aimed at finding and optimizing the design and parameters of the inductor and induction heating installation in general.

Simulation of thermal and electromagnetic processes is based on solving a system of differential equations (Fourier heat equation and Maxwell equations) [13].

COMSOL Multiphysics was chosen as a software product for solving the system of differential equations describing the ongoing processes, which was used to solve problems of this nature.

COMSOL Multiphysics allows to solve the following PDE equation in an arbitrary setting (without using a specific physics interface), which makes it possible to build new models and simulate non-standard processes:

$$
e_{a} \frac{\partial^{2} u}{\partial t^{2}}+d_{a} \frac{\partial u}{\partial t}-\nabla(c \nabla u+\propto u-\gamma)+\beta \nabla u+\alpha u=f
$$

The creation of a mathematical model is due to the need to develop an induction heating technology by a spiral inductor for the production of seamless pipe tees (see Figure 1).

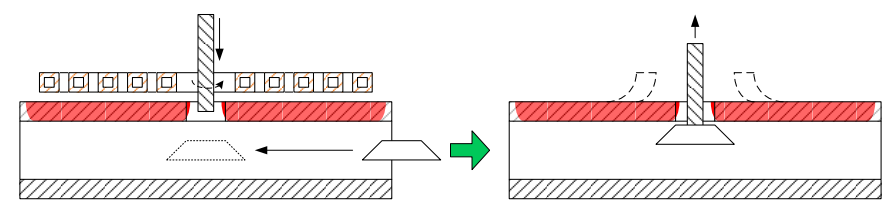

Fig. 1. The technology of seamless tees production.

The fundamental expression for the technological process frequency is determined from the following equation:

$$
\delta=\sqrt{\frac{1}{\pi f \mu \mu_{0} \sigma}}
$$

The most suitable frequency from the industrial frequency range is $1 \mathrm{kHz}$ for the pipe wall thicknesses from $15 \mathrm{~mm}$ to $70 \mathrm{~mm}$. But working at the given frequency (during heating pipes process) leads to resonance and strong noise effect (from practical experience). Therefore, the frequency of $2.4 \mathrm{kHz}$ was chosen as the working frequency.

\section{Mathematical model of induction heating}

The computational domain is shown in Figure 2. 


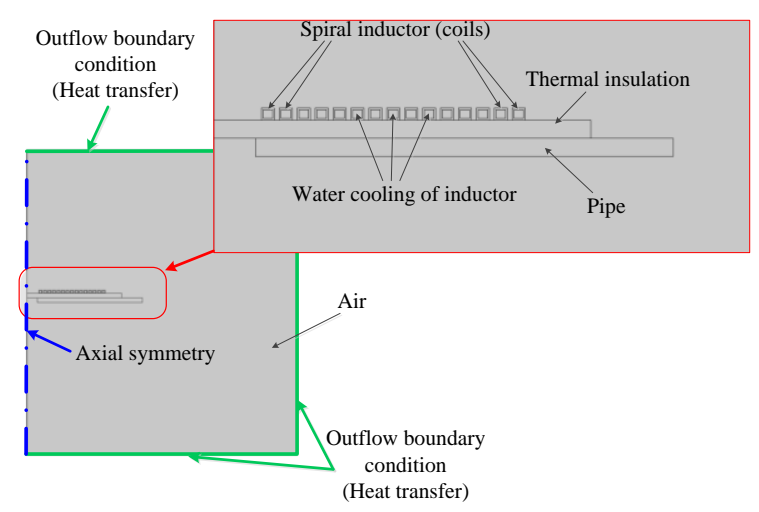

Fig. 2. The computational domain for numerical simulation.

The following assumptions are used in the model:

- Axial symmetry (the geometry of the model is axisymmetric with respect to the axis of the hole, the radius of the pipe truncation is not taken into account).

- Air is considered as a solid medium (there is no convective heat transfer to accelerate the calculation process).

Basic equations of the non-stationary mathematical model for the simulation of induction heating:

- Maxwell's system of equations:

$$
\left\{\begin{array}{c}
\nabla \times \mathbf{H}=\boldsymbol{J} \\
\boldsymbol{B}=\nabla \times \mathbf{A} \\
\boldsymbol{J}=\sigma \boldsymbol{E}+j \omega \boldsymbol{D}+\sigma \boldsymbol{V} \times \boldsymbol{B}+\boldsymbol{J}_{e} \\
\boldsymbol{E}=-j \omega \boldsymbol{A}
\end{array}\right.
$$

where $\sigma=f(T)$ - temperature-dependent electrical conductivity; $\boldsymbol{B}=\mu_{0} \mu \boldsymbol{H} ; \mu=f(H)-$ permeability of workpiece (Steel 08) and $\mu=1$ for other regions of computational domain; $\boldsymbol{J}_{e}$ - external current (feedback with inductor coils).

- "Single-Turn Coil" equations:

- Heat balance equation:

$$
\left\{\begin{array}{c}
I_{c o i l}=\int J e_{c o i l} \\
\boldsymbol{J}_{e}=\sigma \frac{\boldsymbol{V}_{\text {coil }}}{2 \pi r} e_{\text {coil }}
\end{array}\right.
$$

$$
\rho C_{p} \frac{\partial T}{\partial t}-\lambda \nabla T=Q_{s},
$$

where $T$ - temperature; $\rho=f(T)$ - temperature-dependent density; $C_{p}=f(T)-$ temperature-dependent heat capacity; $\lambda=f(T)$ - temperature-dependent thermal conductivity; $Q_{s}$ - heating source density.

- Heat Source (losses of water cooling):

$$
Q_{s w}=G_{w} C_{p}\left(T_{i n}-T\right) /\left(2 \pi r h_{i n} w_{i n}\right)
$$

where $G_{w}$ - water consumption; $T_{i n}$ - inlet water temperature; $T$ - finite element temperature; $r$ - finite element r-coordinate; $h_{i n}$ and $w_{i n}$ - internal parameters of the inductor pipe (height and width of the hole).

\section{Mathematical model of inductor cooling system}

An inductor wire and an individual current-carrying elements of the inductor are heated when the current flows through them. In addition, the heated part radiates heat, some of which is absorbed by the inductor. And the inductor elements must be cooled to protect 
against overheating. There are various methods for calculating the required amount of water for effective cooling $[14,15]$.

The inductor is heated by a current (passing through it) and by losses of the heated pipe through thermal insulation.

The total amount of heat (that heats the inductor):

$$
\Delta P=P_{T}\left(\frac{1}{\eta}-1\right)[k W]
$$

where $P_{T}$ - useful power in workpiece; $\eta$ - total efficiency of the inductor [12].

Quantity of cooling water:

$$
W=\frac{0.24 \Delta P}{T_{2}-T_{1}}\left[\mathrm{~m}^{3} / \mathrm{s}\right],
$$

where $T_{1}$ - water inlet temperature, ${ }^{\circ} \mathrm{C} ; T_{2}$ - water outlet temperature, ${ }^{\circ} \mathrm{C}$ (must be smaller $50^{\circ} \mathrm{C}$ ).

The best cooling is provided by the turbulent water flow [16-18], which is carried out at a sufficient speed of its. This is result of intensive water mixing in an inductor tube. The inequality for turbulent water flow is valid in the inductor tube[19-21]:

$$
\operatorname{Re}=\frac{v D_{0}}{\mu^{\prime}}>2300
$$

where Re - Reynolds number; $v$ - water flow velocity; $\mu^{\prime}=f(T)$ - temperaturedependent kinematic water viscosity (see Table 1 ); $D_{0}$ - hydraulic equivalent of diameter.

Hydraulic equivalent of diameter:

$$
D_{0}=\frac{4 S}{F}
$$

where $S$ - hole area; $F$ - internal perimeter of the pipe.

Table 1. Kinematic water viscosity.

\begin{tabular}{|c|c|c|c|c|c|}
\hline$T,{ }^{\circ} \mathrm{C}$ & 20 & 40 & 60 & 80 & 100 \\
\hline$\mu^{\prime} \cdot 10^{6}, \mathrm{~m}^{2} / \mathrm{s}$ & 1.01 & 0.661 & 0.482 & 0.368 & 0.296 \\
\hline
\end{tabular}

The computational domain of inductor cooling system is shown in Figure 3.

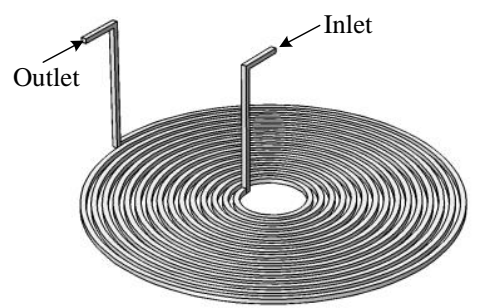

Fig. 3. The computational domain of inductor cooling system for numerical simulation.

The following equations are used for simulation of water flow:

- Momentum equation:

$$
\rho \frac{\partial v}{\partial t}+\rho(\boldsymbol{v} \nabla) \boldsymbol{v}=\nabla\left[-p I+\mu\left(\nabla v+(\nabla v)^{T}\right)\right]
$$

where $I$ - identity matrix; $\mu=f(T)$ - temperature-dependent water viscosity; $\rho=f(T)-$ temperature-dependent water density.

- Continuity equation:

$$
\rho \frac{\partial v}{\partial t}+\nabla(\rho \boldsymbol{v})=0
$$

Water consumption is $2000 \mathrm{~kg} / \mathrm{h}$. Maximum temperature is $1150{ }^{\circ} \mathrm{C}$ and $\Delta T=100{ }^{\circ} \mathrm{C}$ (maximum temperature difference between the outer and the inner surfaces of the pipe) according to the terms of reference. More uniform temperature distribution can be achieved by increasing the heating time [22-24]. 


\section{Results}

One of the main simulation results is the temperature distribution. Volume temperature distribution for the pipe with wall thickness $15 \mathrm{~mm}$ and frequency $2.4 \mathrm{kHz}$ at different time is shown in Figure 4.

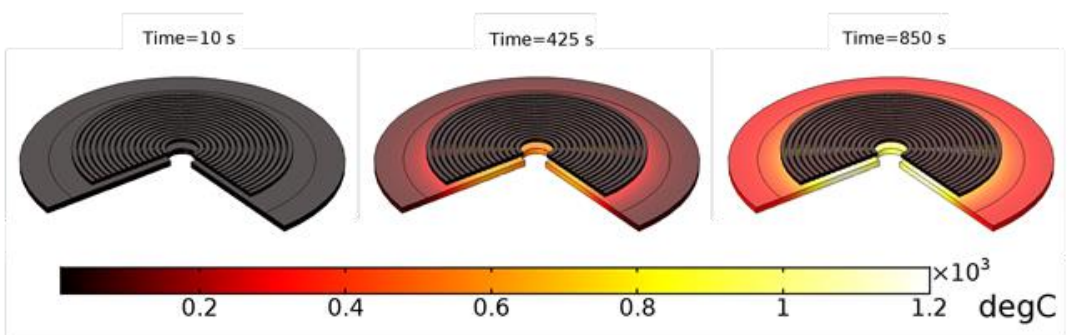

Fig. 4. Volume temperature distribution (wall thickness $15 \mathrm{~mm}$ and frequency $2.4 \mathrm{kHz}$ ).

Heating time is $14 \mathrm{~min} 10 \mathrm{~s}$ for workpiece with wall thickness $15 \mathrm{~mm}$ (for $2.4 \mathrm{kHz}$ ). Temperature difference between the outer and the inner surfaces of the pipe can be visually estimated from Figure 5.

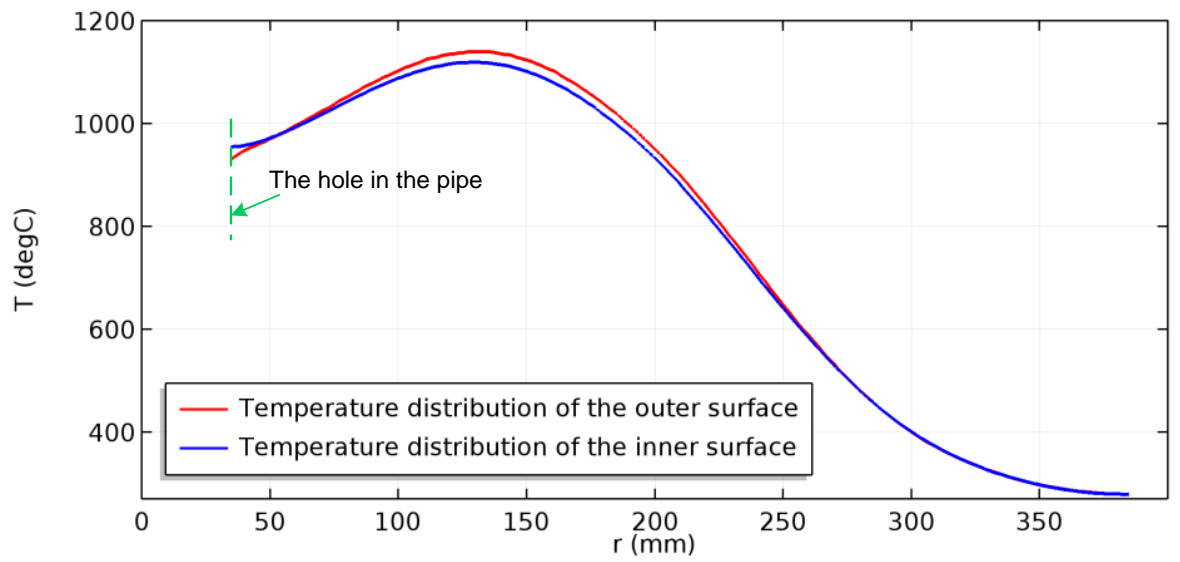

Fig. 5. Temperature distribution of the outer and the inner surfaces of the pipe.

Heating time is shown in Table 2 for different wall thicknesses and frequencies.

Table 2. Heating time.

\begin{tabular}{|c|c|c|c|}
\hline № & Wall thickness, $\mathrm{mm}$ & Heating time for $1 \mathrm{kHz}, \mathrm{s}$ & Heating time for $2.4 \mathrm{kHz}, \mathrm{s}$ \\
\hline 1 & 15 & 650 & 850 \\
\hline 2 & 40 & 1220 & 1670 \\
\hline 3 & 60 & 2080 & 2810 \\
\hline 4 & 70 & 2110 & 2870 \\
\hline
\end{tabular}

Velocity distribution of liquid for inductor cooling system is shown in Figure 6. According to the simulation results, the specified cooling system parameters meet the requirements for effective heat removal. The steady state is observed after $1 \mathrm{~s}$ after the water supply and the differential pressure is $0.5 \mathrm{~atm}$. 


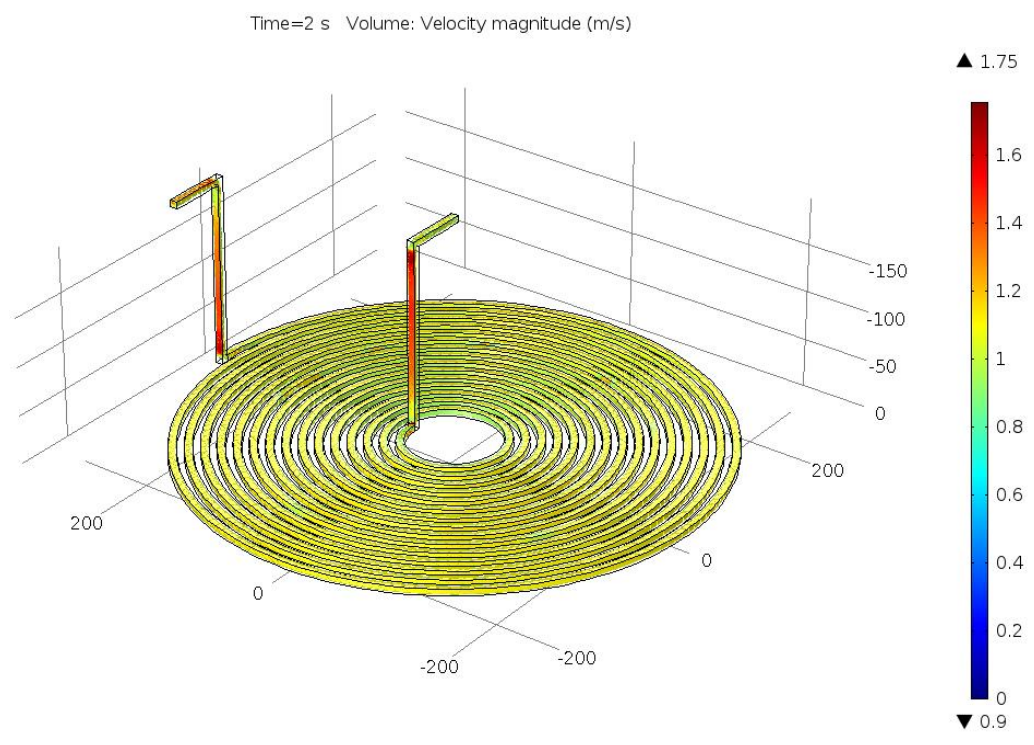

Fig. 6. Velocity distribution of liquid inside inductor.

\section{Verification of mathematical model}

A series of experimental studies by measuring the temperature at the inner surface of the pipe (wall thickness $15 \mathrm{~mm}$ ) were performed for verification of the mathematical model. The installation for carrying out experimental research was made in VNIITVCh. The technology process parameters were taken from simulation. The scheme of the experimental study is shown in Figure 7. The temperature was measured by a pyrometer along the line (along the axis) of the pipe. The graph also shows the measurement error.

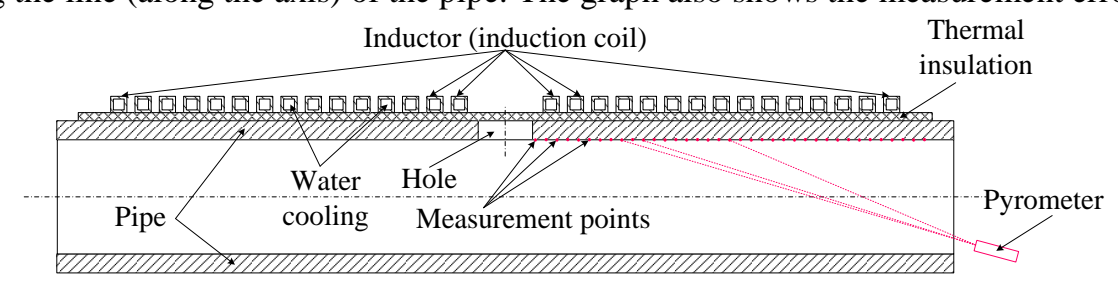

Fig. 7. The scheme of the experimental study.

The comparison (experimental data and simulation results) of temperature distribution of the inner surface of the pipe is shown in Figure 8. 


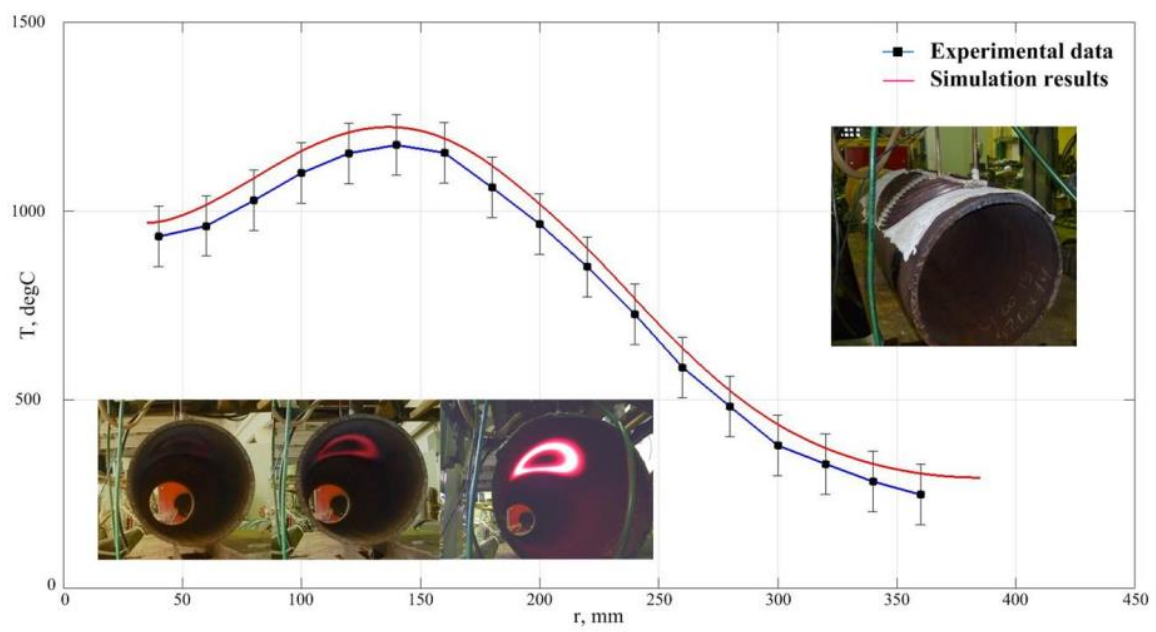

Fig. 8. The comparison of temperature distribution.

The process of the workpiece heating with a thickness of $15 \mathrm{~mm}$ takes $\approx 14$ minutes. The results of the simulation are confirmed by the results of the experimental study, which allows to verify the developed mathematical model. The developed mathematical model allows to calculate geometrical and electrical parameters of the induction heating equipment without additional experimental studies.

\section{Conclusions}

The mathematical model of induction heating process for technology of tees production is developed and verified according to the results of experimental studies. The technology process and power source parameters were taken from simulation. The developed mathematical model allows to determine optimal operating modes by simulation results without additional experimental studies.

The inductor cooling system parameters were taken from the developed hydrodynamic 3D mathematical model. According to the results of simulation $\mathrm{Re} \gg 2300$ (the best cooling is provided by the turbulent water flow).

The capacity of the capacitor bank and the construction of the inductors are determined from the simulation results. The developed technology of making seamless tees allowed to increase the productivity of the manufacturing process several times.

\section{References}

1. A. Muhlbauer, History of induction heating and melting. (2008)

2. M. Shevtsov, A. Borodachev, Development of electrothermal technique. (1983)

3. A. Muhlbauer, Historical overview of induction melting and heating, Proc. Int. Conf. on Heating by Electromagnetic Sources HES-04. (2001)

4. T. Tanaka, K. Kuroda, A. Kuroda, ISIJ Int., 32, 575 (1992)

5. C. Dodd, Journal of Applied Physics, 39(6), 2829-2838 (1968)

6. A. Fujita, H. Sadakata, I. Hirota, H. Omori, M. Nakaoka, IEEE IPEMC'09. 3. 25372544 (2009) 
7. A. Lisenkov, V. Frolov, S. Dresvin, Electrotechnological industrial equipment. (2010)

8. V. Bodazhkov, Induction heating of pipes. (1969)

9. A. Slukhotsky, N. Pavlov, Industrial application of high frequency currents, 7, 8-29 (1966)

10. A. Ogorodnikov, Modeling in the MatLab-COMSOL 3.5a environment. (2012)

11. S. Dresvin, N.K. Shi, D. Ivanov, J. Amouroux, High Temperature Material Processes, 15(1), 7-13 (2011)

12. N. Obraztsov, V. Frolov, 2018 IEEE Conference of Russian Young Researchers in Electrical and Electronic Engineering (EIConRus). (2018)

13. V. Nemkov, B. Polevodov, S. Gurevich, Mathematical modeling of high-frequency heating devices. (1991)

14. A. Slukhotsky, S. Ruskin, Inductors for induction heating. (1974)

15. P. Di Barba, F. Dughiero, A. Savini, IEEE Transactions on Magnetics, 39(3), 15191522 (2003)

16. I. Murashov, The development of dc plasma torch for the spraying technology taking into account the phenomena of plasma flow instability. (2016)

17. N. Zheng, P. Liu, F. Shan, Zh. Liu, W. Liu, International Journal of Thermal Sciences, 111, 289-300 (2017)

18. Pranit M. Patil, Amol P. Yadav, P. Patil, IJIRSET, 4(4), 2223-2226 (2015)

19. F. C. K. Ting, J. T. Kirby, Coastal Engineering, 27(3-4), 131-160 (1996)

20. Y. Jin, M.-F. Uth, A. Kuznetsov, H. Herwig, Journal of Fluid Mechanics, 766, 76-103 (2015)

21. K. Avila, D. Moxey, A. de Lozar, M. Avila, D. Barkley, B. Hof, Science, 333(6039), 192-196 (2011)

22. N. Glukhanov, Physical basis of high-frequency heating (1979)

23. R. Sabariego, P. Sergeant, J. Gyselinck, P. Dular, L. Dupre, and J. Melkebeek, IEEE Transactions on Magnetics, 42(4), 1407-1410 (2006)

24. P. Alotto, A. Spagnolo, and B. Paya, IEEE Transactions on Magnetics, 47(5), 12701273 (2011) 